\title{
Diamagnetic and paramagnetic Meissner effect from odd-frequency pairing in multiorbital superconductors
}

\author{
Fariborz Parhizgar $\odot$ and Annica M. Black-Schaffer $\odot$ \\ Department of Physics and Astronomy, Uppsala University, Box 516, S-751 20 Uppsala, Sweden
}

(Received 22 March 2021; revised 11 June 2021; accepted 28 July 2021; published 9 August 2021)

\begin{abstract}
The Meissner effect is one of the defining properties of superconductivity, with a conventional superconductor completely repelling an external magnetic field. In contrast to this diamagnetic behavior, odd-frequency superconducting pairing has often been seen to produce a paramagnetic Meissner effect, which instead makes the superconductor unstable due to the attraction of magnetic field. In this paper, we study how both even- and odd-frequency superconducting pairing contributes to the Meissner effect in a generic two-orbital superconductor with a tunable odd-frequency pairing component. By dividing the contributions to the Meissner effect into intraand interband processes, we find that the odd-frequency pairing actually generates both dia- and paramagnetic Meissner responses, determined by the normal-state band structure. More specifically, for materials with two electronlike (holelike) low-energy bands, we find that the odd-frequency interband contribution is paramagnetic but nearly canceled by a diamagnetic odd-frequency intraband contribution. Combined with a diamagnetic even-frequency contribution, such superconductors thus always display a large diamagnetic Meissner response to an external magnetic field, even in the presence of large odd-frequency pairing. For materials with an inverted, or topological, band structure, we find the odd-frequency interband contribution to instead be diamagnetic and even the dominating contribution to the Meissner effect in the near-metallic regime. Taken together, our results show that odd-frequency pairing in multiorbital superconductors does not generate a destabilizing paramagnetic Meissner effect and can even generate a diamagnetic response in topological materials.
\end{abstract}

DOI: 10.1103/PhysRevB.104.054507

\section{INTRODUCTION}

Odd-frequency superconductivity is an unusual superconducting state where the two electrons forming a Cooper pair join each other at different times, such that the resulting pair amplitude is odd under the interchange of the internal time coordinate or, equivalently, has an odd frequency dependence [1-4]. The oddness under time interchange has direct consequences for the other symmetry properties of the superconducting state, since the fermionic nature of the pair amplitude requires it to be odd under a full interchange of all the electron quantum numbers. For example, the conventional superconducting spin-singlet $s$-wave state can be converted into a spin-triplet $s$-wave state if the frequency dependence becomes odd. This particular situation has been shown to arise when a conventional superconductor is in proximity with a ferromagnet, where the spin-triplet pairing allows for a longrange proximity effect into the ferromagnet [4-6].

More recently, odd-frequency superconductivity was also shown to occur in materials with an additional low-energy degree of freedom [7], such as multiorbital or band [8,9],

Published by the American Physical Society under the terms of the Creative Commons Attribution 4.0 International license. Further distribution of this work must maintain attribution to the author(s) and the published article's title, journal citation, and DOI. Funded by Bibsam. double dot [10,11], or bilayer structures [12]. In these systems, the orbital (or similar) degree of freedom adds an additional index to the pair amplitude, such that it is, e.g., possible to have odd-frequency pairing in the form of spin-singlet $s$-wave odd interorbital pairing. Notably, this odd-frequency pairing easily occurs in the bulk and does not require any spatial inhomogeneity in form of junctions or similar. The condition to generate odd-frequency pairing in a multiorbital superconductor [7,8] is, in fact, easily fulfilled as it only requires finite interorbital (single particle) hybridization and that the strength of the superconducting pairing is different in different orbitals, also known as a pairing asymmetry or orbital selectivity [13]. As such, many known multiband superconductors have recently been shown to host odd-frequency pairing, including doped topological insulators [9,14], $\mathrm{Sr}_{2} \mathrm{RuO}_{4}$ [15], $\mathrm{UPt}_{3}$ [16], and superconducting Weyl semimetals [17,18]. Based on these results, even iron-based superconductors with orbital selective pairing [13] likely host odd-frequency pairing.

As odd-frequency pairing is intrinsically time dependent, it has so far been hard to detect directly, becoming essentially a hidden dynamic order. However, for specific systems, signatures of odd-frequency pairing have been found in experimentally accessible quantities, such as the existence of finite density of states at zero energy [19], Kerr effect $[15,16]$, or Josephson current in junctions where even-frequency superconductivity becomes forbidden [17,20-22]. Most prominent of the odd-frequency signatures is probably, however, the prediction of a paramagnetic Meissner effect [23-30]. 
The Meissner effect is the response of a superconductor to a weak magnetic field, with a conventional superconductor always completely repelling the magnetic field, known as a diamagnetic Meissner effect [31]. In contrast to this conventional diamagnetic response, odd-frequency pairing has instead in many circumstances been predicted to cause a paramagnetic Meissner effect, where the superconductor instead attracts a magnetic field, which then ultimately destabilizes the whole superconducting state by enhancing the magnetic field inside the superconductor [23-30]. This paramagnetic response has recently been experimentally confirmed in regions where odd-frequency superconductivity is proximity-induced in various heterostructures [27,30]. In these heterostructures, the paramagnetic response is not a problem, since superconductivity is originating from a conventional bulk superconductor that still has a diamagnetic Meissner effect and is thus stable in the presence of a weak magnetic field. However, finding a paramagnetic Meissner effect in the bulk of a superconductor directly raises issues of overall stability of the superconducting phase. In fact, the existence of a paramagnetic Meissner effect has been used as a key argument against the existence of an intrinsic bulk odd-frequency superconducting state $[28,32,33]$.

The issue with a possible paramagnetic Meissner effect from odd-frequency pairing causes a clear conundrum for odd-frequency superconductivity in multiorbital superconductors, as the odd-frequency pairing is here clearly a bulk effect and therefore directly raises the question of how stable these superconductors actually are. Previously, we have shown that for a very unconventional superconductor, the $(\mathrm{Cu}-)$ doped $\mathrm{Bi}_{2} \mathrm{Se}_{3}$ topological insulator, odd-frequency pairing can indeed produce a small, but still, diamagnetic Meissner response [9] that produces a counterexample of the expectation of paramagnetic Meissner effect from oddfrequency pairing. However, this is a very special situation, as doped $\mathrm{Bi}_{2} \mathrm{Se}_{3}$ has a linear band dispersion with exceptionally strong spin-orbit coupling and a highly unconventional superconducting state consisting of spin-triplet interorbital pairing that generates a nematic superconducting state. In this paper, we tackle the much more general problem of the relation between odd- and even-frequency pairing and Meissner effect in a generic two-orbital bulk superconductor with a standard normal-state quadratic dispersion and conventional $s$-wave superconductivity. By dividing up the contributions to the Meissner effect from the even- and odd-frequency pairing and also from intra- and interband processes, we are able to derive simple analytical expressions that show that odd-frequency pairing generates both diamagnetic (positive) and paramagnetic (negative) Meissner contributions in these generic systems. In particular, the intra- and interband oddfrequency contributions always have different signs, with the signs determined by the normal-state band structure.

More specifically, our results show that for a material with two electronlike quadratically dispersive bands (or holelike), see Fig. 1(a), we find that the odd-frequency interband Meissner contribution is paramagnetic, but it is always compensated by an almost equally large but diamagnetic intraband contribution. Adding to that an overall diamagnetic contribution from the even-frequency pairing, the total Meissner effect of the superconductor is always diamagnetic, even in the limit of

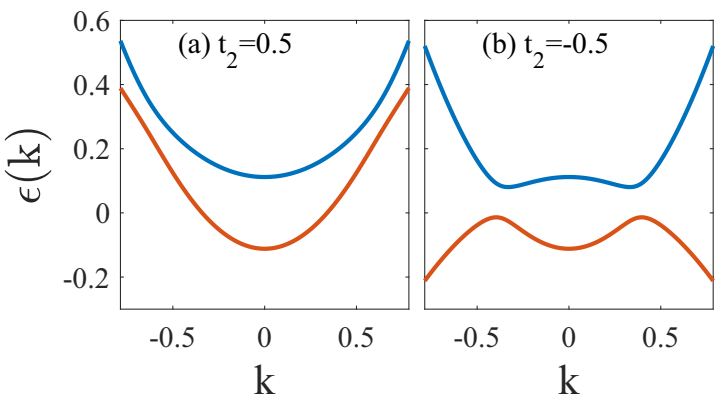

FIG. 1. Normal state band structure given by Eq. (1) for a two-orbital superconductor for (a) electronlike band structure with $t_{2}=0.5$ and (b) inverted band structure with $t_{2}=-0.5$. Here $t_{12}=0.05, \mu=0, \delta \mu=-0.2$.

large odd-frequency pairing. On the other hand, for a material with an inverted band structure, i.e., one electronlike and one holelike band, still with quadratic dispersion, as found in topological materials, see Fig. 1(b), odd-frequency pairing instead generates a diamagnetic inter-band contribution to the Meissner effect that is always larger than the intraband paramagnetic contribution. Very interestingly, in the near-metallic regime, the odd-frequency interband contribution becomes dominating and thus the total diamagnetic Meissner effect of the superconductor is even driven by the odd-frequency pairing. These results show that odd-frequency pairing in multiorbital superconductors does not produce a destabilizing paramagnetic Meissner effect but can even generate a diamagnetic response in topological materials.

The organization of the rest of the paper is as follows. In Sec. II, we first present the Hamiltonian for a generic twoorbital superconductor and then derive its superconducting pair amplitudes in Sec. II A and the general theory and calculational details for the Meissner effect in Sec. II B. We then present our results in Sec. III, both analytical and numerical, divided up into two different cases: when the resulting energy bands have the same signs of their curvatures, both being electronlike, in Sec. III A, and when they are inverted, or topological, with different signs of their curvatures, in Sec. III B). Finally, we summarize our results in Sec. IV. We also provide some more details of the calculations in Appendixes A and B.

\section{THEORY}

\section{A. Generic two-orbital superconductor}

To investigate odd-frequency superconductivity and Meissner effect in multiorbital superconductors, we assume a bulk material with a two generic low-energy orbitals in the normal state. This is the simplest model that leads to the presence of bulk odd-frequency superconducting pairing. The normal state is described in the orbital basis as $h(k)=\xi_{+} \tau_{0}+\xi_{-} \tau_{z}+$ $\xi_{12} \tau_{x}$, where $\xi_{1(2)}=\xi_{+} \pm \xi_{-}$and $\xi_{12}$ represent the intra- and interorbital kinetic energy dispersions, respectively. Here, $\tau$ represents the Pauli matrices in the orbital basis and we assume the Hamiltonian to be spin degenerate. Diagonalizing this normal part results in the two energy bands:

$$
\epsilon_{ \pm}=\xi_{+} \pm \sqrt{\xi_{-}^{2}+\xi_{12}^{2}}
$$


To make our work general, yet simple enough to allow a partial analytical treatment, we assume the dispersion relations in two orbitals to be of the form $\xi_{1(2)}=-\mu+t_{1(2)} k^{2}-$ $(-1)^{1(2)} \delta \mu / 2$, with chemical potential $\mu$, orbital energy difference $\delta \mu$, electron wave vector $k$, and effective curvature (the inverse of the effective mass) $t_{1,2}$. For the numerical results we limit ourselves to a two-dimensional wave vector to keep the momentum integrations feasible, but we expect no significant changes for three dimensions. Moreover, we mainly assume a $k$-independent hybridization $\xi_{12}=t_{12}$ between the two orbitals, but we report complementary results for $k$-dependent hybridization $\xi_{12}(k)$ in Appendix A. Throughout this paper, we set the energy scale through $t_{1}=1$ and to investigate the effects of the band structure, we separately treat two cases, $t_{2}>0$ and $t_{2}<0$. In Fig. 1, we plot the band structure given by Eq. (1) for the two different cases, where in (a) $t_{2}=0.5$ and (b) $t_{2}=-0.5$. Thus, in Fig. 1(a), we have two parabolic bands with similar electronlike dispersions but different curvatures with their energy minima at $\pm \sqrt{t_{12}^{2}+(\delta \mu / 2)^{2}}$. In contrast, in Fig. 1(b), we get a so-called inverted band structure, consisting originally of overlapping electron- and holelike bands, which hybridize and form a gapped band structure, such that the Fermi level never crosses both bands. This second model has a band structure closely resembling that of topological insulators and other nontrivial topological materials, but here without any spin-orbit coupling to more clearly elucidate the effect of the band dispersion without unnecessary complications.

We include superconductivity in the system by introducing a conventional spin-singlet intraorbital pairing in each orbital, written as $\hat{\Delta}=\left(\delta_{+} \tau_{0}+\delta_{-} \tau_{z}\right) \otimes i \sigma_{y}$, with $\sigma$ being the Pauli matrices in spin space. Here the spin-singlet order parameter in each orbital is $\delta_{1(2)}=\delta_{+}+(-) \delta_{-}$, which is the simplest possible implementation of superconductivity in a two-orbital model. As we will explicitly see later, odd-frequency pairing is always present in this model for a finite $\delta_{-}$, as long as also $\xi_{12}$ is finite. The other alternative route to generate oddfrequency pairing is to assume a finite inter-orbital pairing $\delta_{12}[7,15]$, but such term can always be described within a change of basis of our current model, see Appendix B, and thus it cannot qualitatively change our results. We also note that throughout this paper we assume that all order parameters are fully tunable and we do not treat them self-consistently. A self-consistent treatment of superconductivity would require us to instead fix (or tune) the pair potential, and as such we can always find setups with the same order parameters as we use here, resulting in the same end result. Moreover, as we are interested in how the Meissner effect varies with varying even- and odd-frequency pairing, it is more beneficial to allow full tuning of the order parameters to explore all realistic possibilities. Finally, writing the full Hamiltonian in the orbital basis $\psi^{\dagger}=\left(c_{1 \uparrow}^{\dagger} c_{2 \uparrow}^{\dagger} c_{1 \downarrow} c_{2 \downarrow}\right)$, we arrive at

$$
\mathcal{H}=\left(\xi_{+} \tau_{0}+\xi_{-} \tau_{z}+\xi_{12} \tau_{x}\right) \otimes \gamma_{z}+\left(\delta_{+} \tau_{0}+\delta_{-} \tau_{z}\right) \otimes \gamma_{x}
$$

where $\gamma$ s are Pauli matrices in the particle-hole basis. The energy eigenvalues of the full Hamiltonian, $\mathcal{H}$, are equal to

$$
\begin{aligned}
\varepsilon_{ \pm}^{2}= & \xi_{+}^{2}+\xi_{-}^{2}+\xi_{12}^{2}+\delta_{+}^{2}+\delta_{-}^{2} \\
& \pm 2 \sqrt{\left(\delta_{-} \delta_{+}+\xi_{-} \xi_{+}\right)^{2}+\left(\delta_{-}^{2}+\xi_{+}^{2}\right) \xi_{12}^{2}}
\end{aligned}
$$

\section{Superconducting pair amplitude}

To understand superconducting pairing in our generic two-orbital superconductor, Eq. (2), we extract the Green's function of the system:

$$
\mathcal{G}=\left(\omega \pm i 0^{+}-\mathcal{H}\right)^{-1} \equiv\left(\begin{array}{cc}
G & F \\
\bar{F} & \bar{G}
\end{array}\right) .
$$

Here, $G$ and $\bar{G}$ represent the electron and hole propagators, respectively, while $F$ and $\bar{F}^{\dagger}=F$ are the anomalous Green's functions or, equivalently, the superconducting pair amplitude. Here $\pm i 0^{+}$is added to the real frequency, $\omega$, to avoid divergencies, with the sign referring to the retarded/advanced Green's functions for $\omega>0$ and $\omega<0$, respectively. The anomalous Green's function can further be decomposed into its even- and odd-frequency components $F=F^{e}+F^{o}$. For the Hamiltonian given in Eq. (2), we find (in the orbital basis)

$$
F^{e}=\frac{1}{D}\left(\begin{array}{cc}
\omega^{2}\left(\delta_{+}+\delta_{-}\right)-\alpha_{+} & 2 \xi_{12}\left(\delta_{+} \xi_{+}-\delta_{-} \xi_{-}\right) \\
2 \xi_{12}\left(\delta_{+} \xi_{+}-\delta_{-} \xi_{-}\right) & \omega^{2}\left(\delta_{+}-\delta_{-}\right)-\alpha_{-}
\end{array}\right)
$$

and

$$
F^{o}=\frac{1}{D}\left(\begin{array}{cc}
0 & -2 \omega \xi_{12} \delta_{-} \\
2 \omega \xi_{12} \delta_{-} & 0
\end{array}\right) .
$$

Here, $\quad \alpha_{ \pm}=\left(\delta_{+} \pm \delta_{-}\right)\left(\xi_{+} \mp \xi_{-}\right)^{2}+\left(\delta_{+} \mp \delta_{-}\right)\left(\xi_{12}^{2}+\delta_{+}^{2}-\delta_{-}^{2}\right)$, while denominator $D=\left(\left(\omega \pm i 0^{+}\right)^{2}-\varepsilon_{+}^{2}\right)\left(\left(\omega \pm i 0^{+}\right)^{2}-\varepsilon_{-}^{2}\right)$, thus being an even function of frequency. Note that we have here dropped the $i 0^{+}$term from $\omega$ in the numerators of Eqs. (5) and (6) as it has no effect on the numerical results.

The even-frequency pair amplitude in Eq. (5) includes both intraorbital (diagonal components) and interorbital (offdiagonal components) components, where the latter is directly proportional to the interorbital hybridization, $\xi_{12}$. The oddfrequency pair amplitude Eq. (6) has only inter-orbital components and is a consequence of the existence of superconducting orbital selectivity, i.e., a finite $\delta_{-}$and finite interorbital hybridization, $\xi_{12}$, i.e., there must exist an asymmetry in the superconducting pairing between the two orbitals, or an orbital selectivity, together with a finite hybridization between the two orbitals for odd-frequency pairs to exist. In addition to generating odd-frequency pairs, the orbital selectivity term $\delta_{-}$causes the superconducting part of the Hamiltonian to not commute with the normal part. This incompatibility between the normal and superconducting parts has recently also been used to define the concept of superconducting fitness [34], which has been shown to be directly linked to the existence of odd-frequency pairs [7]. We can here explicitly verify that all pair amplitudes satisfy the fermionic nature of superconductivity: the interorbital pairs are even (odd) under the interchange of the orbital index when they have an even- (odd-) frequency dependence, as required for spin-singlet pairs with no $k$ dependence ( $s$-wave symmetry). Above, we explicitly worked in the orbital basis for the form 
of the Hamiltonian in the band basis, where the kinetic energy is diagonal, see Appendix B.

\section{B. Meissner effect}

The Meissner effect is the response of a superconductor to an externally applied magnetic field. Within linear response theory, the current response function $\mathbf{j}$ to the vector potential of the magnetic field $\mathbf{A}$ can be obtained via $j_{\mu}(\mathbf{q}, \Omega)=$ $-K_{\mu \nu}(\mathbf{q}, \Omega) A_{v}(\mathbf{q}, \Omega)$. Here, $K$ is the current-current correlation function and $\nu, \mu$ are the directions of the applied vector potential and current response, respectively, while $\mathbf{q}, \Omega$ are the wave vector and frequency of the response function. The Meissner response is obtained for a static and uniform magnetic field, thus taking the limits $\Omega \rightarrow 0$ and $\mathbf{q} \rightarrow 0$, in the order. To calculate $K$, we need the current operator matrix $\mathcal{J}=\mathcal{J}_{\mu}^{p}+\mathcal{J}_{\mu \nu}^{d} A_{\nu}$, divided into its paramagnetic and diamagnetic part and which we find in the standard way by first introducing the vector potential $\mathbf{A}$ into the Hamiltonian through the substitution $\mathbf{k} \rightarrow \mathbf{k}-\mathbf{A}$ and then taking the derivative with respect to $A_{\nu}$. The Meissner response can then be written as $[9,35]$

$$
K_{\mu \nu}(\mathbf{q}=0, \Omega=0)=-\sum_{\omega} \sum_{\mathbf{k}} \operatorname{Tr}\left\{\mathcal{G J}_{\mu}^{p} \mathcal{G J}_{\nu}^{p}+\mathcal{G J}_{\mu \nu}^{d}\right\}
$$

where $\mathcal{G}$ is the Green's function. We here work in natural units, such that we set $\hbar=c=e=m=1$. Moreover, since our system is isotropic in space, we can without loss of generality focus on a specific direction, say the $x$ direction, of the Meissner response function and thus drop the $\mu, v$ indices. Finally, using the fact that the current operator $\mathcal{J}^{p(d)}$ in the particle-hole basis takes the diagonal form $\left(\begin{array}{cc}J^{p(d)} & 0 \\ 0 & \bar{J}^{p(d)}\end{array}\right)$, the Meissner response in Eq. (7) can be simplified as

$$
K=-2 \sum_{\omega} \sum_{\mathbf{k}} \operatorname{Tr}\left\{G J^{p} G J^{p}+F \bar{J}^{p} \bar{F} J^{p}+G J^{d}\right\} .
$$

Since the focus of this paper is to obtain the contributions of the odd- and even-frequency pairing to the Meissner effect, we only need to focus on the second term of the above equation,

$$
K_{F}=-\sum_{\omega} \sum_{\mathbf{k}} \operatorname{Tr}\left\{F \bar{J}^{p} \bar{F} J^{p}\right\},
$$

because only this term depends on the pair amplitudes $F, \bar{F}$ [9,36]. Our interest is thus focused on the Meissner kernel of Eq. (9): $\mathcal{K}=\operatorname{Tr}\left\{F \bar{J}^{p} \bar{F} J^{p}\right\}$. This term can always be decomposed into $\mathcal{K}=\mathcal{K}^{e}+\mathcal{K}^{o}$, where $\mathcal{K}^{e}=\operatorname{Tr}\left\{F^{e} \bar{J}^{p} \bar{F}^{e} J^{p}\right\}$ is entirely due to the even-frequency pairing, while $\mathcal{K}^{o}=$ $\operatorname{Tr}\left\{F^{o} \bar{J}^{p} \bar{F}^{o} J^{p}\right\}$ comes exclusively from the odd-frequency pairing. Technically, there also exists terms of the form $F^{o} \bar{J}^{p} \bar{F}^{e} J^{p}$ in $\mathcal{K}$ but, since they are always odd in frequency, they automatically cancel during the final frequency summation in Eq. (8).

\section{Kernel decomposition into intra- and interband processes}

To better understand the physics of the Meissner response, we decompose its kernel $\mathcal{K}$ into parts coming from inter- and intraband processes, respectively, as is often beneficial for response functions. Using the fact that the Meissner kernel can generically be written as $\mathcal{K}=\left(a \omega^{4}+2 b \omega^{2}+c\right) / D^{2}$, we can form the following decomposition:

$$
\mathcal{K}=\frac{\mathcal{K}_{+}}{\left(\omega^{2}-\varepsilon_{+}^{2}\right)^{2}}+\frac{\mathcal{K}_{-}}{\left(\omega^{2}-\varepsilon_{-}^{2}\right)^{2}}+\frac{\mathcal{K}_{12}}{\left(\omega^{2}-\varepsilon_{+}^{2}\right)\left(\omega^{2}-\varepsilon_{-}^{2}\right)} .
$$

Here $\mathcal{K}_{ \pm}$and $\mathcal{K}_{12}$ are frequency-independent coefficients for processes involving only one individual band (i.e., intraband processes) and both bands (i.e., interband processes), respectively. These coefficients are given by

$$
\begin{aligned}
\mathcal{K}_{ \pm} & =\frac{a \varepsilon_{ \pm}^{4}+2 b \varepsilon_{ \pm}^{2}+c}{\left(\varepsilon_{+}^{2}-\varepsilon_{-}^{2}\right)^{2}} \\
\mathcal{K}_{12} & =-2 \frac{a \varepsilon_{+}^{2} \varepsilon_{-}^{2}+b\left(\varepsilon_{+}^{2}+\varepsilon_{-}^{2}\right)+c}{\left(\varepsilon_{+}^{2}-\varepsilon_{-}^{2}\right)^{2}} .
\end{aligned}
$$

We here note that the intraband term is the only term appearing in a single-orbital superconductor, or for a two-orbital superconductor in the trivial $\xi_{12}=0$ limit, while the interband term only appears when $\xi_{12}$ is finite. We also emphasize here that these are processes between different bands, i.e., the eigenstates of the Hamiltonian Eq. (2) and not processes between the different orbitals.

\section{Frequency summation}

To analytically perform the summation over frequency in the Meissner kernel in Eq. (9), we use analytical continuation from real frequency to Matsubara frequency, $\omega \rightarrow i \omega_{n}$. Then, by applying Matsubara frequency summation, the Meissner response reads

$$
\begin{aligned}
K_{F}= & \sum_{\mathbf{k}} \frac{\mathcal{K}_{+}}{2 \varepsilon_{+}^{2}}\left(C\left(\varepsilon_{+}\right)+n^{\prime}\left(\varepsilon_{+}\right)\right)+\frac{\mathcal{K}_{-}}{2 \varepsilon_{-}^{2}}\left(C\left(\varepsilon_{-}\right)+n^{\prime}\left(\varepsilon_{-}\right)\right) \\
& +\mathcal{K}_{12} \frac{C\left(\varepsilon_{-}\right)-C\left(\varepsilon_{+}\right)}{\varepsilon_{+}^{2}-\varepsilon_{-}^{2}}
\end{aligned}
$$

where $n(\xi)$ is the Fermi-Dirac distribution function, $n^{\prime}(\xi)$ its derivative with respect to energy, and $C(\xi)=(n(-\xi)-$ $n(\xi)) / 2 \xi$. Here we note that both the $C(\varepsilon)$ and $\frac{C\left(\varepsilon_{-}\right)-C\left(\varepsilon_{+}\right)}{\varepsilon_{+}^{2}-\varepsilon_{-}^{2}}$ functions are always positive, while $n^{\prime}(\varepsilon)$ is always negative. Moreover, assuming we always have a gapped superconductor, as is usually the case for the bulk of $s$-wave states, the $n^{\prime}(\varepsilon)$ term is negligible, and hence it is the signs of $\mathcal{K}_{ \pm}$and $\mathcal{K}_{12}$ that become the decisive factors for determining the sign of the Meissner effect.

We can further simplify the expression for the Meissner effect by noting that, if we have a $k$-independent orbital hybridization $\xi_{12}$ as we assume here in the main text, the current operator is diagonal in the orbital basis: $J^{p}=\left(\begin{array}{cc}j_{1} & 0 \\ 0 & j_{2}\end{array}\right)$ and similarly for $\bar{J}^{p}=-J^{p}$ (very results for a $k$-dependent hybridization are reported in Appendix A). Moreover, considering that the anomalous Green's function takes the form $\left(\begin{array}{ll}f_{1} & f_{12} \\ f_{21} & f_{2}\end{array}\right)$, the Meissner kernel can be written,

$$
\mathcal{K}=j_{1}^{2} f_{1}^{2}+j_{2}^{2} f_{2}^{2}+2 j_{1} j_{2} f_{12} f_{21}=\left(a \omega^{4}+2 b \omega^{2}+c\right) / D^{2},
$$


where the last equality is just iterating the statement from before used to parametrize the Meissner kernel. Here, the first two terms in the first expression are always positive, while the last term changes signs based on the sign of $j_{1} j_{2}$ and $f_{12} f_{21}$. Since $j_{1(2)}=-\partial \xi_{1(2)}(k-A) / \partial A=2 t_{1(2)} k$, the sign of the product $j_{1} j_{2}$ is equivalent to the sign of $t_{2}$, since we set $t_{1}=1$. It is thus natural when studying the Meissner effect to distinguish between the two cases: $t_{2}>0$ giving $j_{1} j_{2}>0$, which has two bands with the same curvature, and $t_{2}<0$ with $j_{1} j_{2}<0$, which has an inverted band structure. This is particularly important for the case of the odd-frequency pairing contribution to the Meissner effect as this pairing contains only the interorbital terms $f_{12}, f_{21}$.

\section{RESULTS}

Having established the underlying theory, we can now focus on calculating the Meissner effect in generic two-orbital superconductors described by Eq. (2). Thanks to the derivations in the preceding section, we can proceed analytically to a large degree. To calculate the Meissner kernel in terms of its intra- and interband contributions in Eq. (10), we need the coefficients $a, b, c$ in Eq. (11). For a $k$-independent orbital hybridization $\xi_{12}$ these are directly accessible using Eq. (13). For the contribution to the Meissner kernel from the evenfrequency pairing, we use Eq. (5) for the anomalous Green's function components and arrive at the coefficients:

$$
\begin{aligned}
& a^{e}=j_{1}^{2} \delta_{1}^{2}+j_{2}^{2} \delta_{2}^{2}, \\
& b^{e}=-\left(j_{1}^{2} \alpha_{+} \delta_{1}+j_{2}^{2} \alpha_{-} \delta_{2}\right), \\
& c^{e}=j_{1}^{2} \alpha_{+}^{2} \delta_{1}+j_{2}^{2} \alpha_{-}^{2}+2 j_{1} j_{2} f_{12}^{2} .
\end{aligned}
$$

These, plugged in Eq. (11), directly give the even-frequency Meissner kernel contributions $\mathcal{K}_{ \pm}^{e}$ and $\mathcal{K}_{12}^{e}$. For the contributions to the Meissner kernel from the odd-frequency pairing, we instead use Eq. (6) and find that $a^{o}=c^{o}=0$, which means we can straightforwardly simplify the expressions to arrive at

$$
\begin{aligned}
\mathcal{K}_{ \pm}^{o} & =j_{1} j_{2} \frac{8 \delta_{-}^{2} \xi_{12}^{2} \varepsilon_{ \pm}^{2}}{\left(\varepsilon_{+}^{2}-\varepsilon_{-}^{2}\right)^{2}}, \\
\mathcal{K}_{12}^{o} & =-j_{1} j_{2} \frac{8 \delta_{-}^{2} \xi_{12}^{2}\left(\varepsilon_{-}^{2}+\varepsilon_{+}^{2}\right)}{\left(\varepsilon_{+}^{2}-\varepsilon_{-}^{2}\right)^{2}} .
\end{aligned}
$$

These equations show that for odd-frequency pairing, the sign of the intra- and interband Meissner kernels, and thus their contributions to the Meissner effect, are opposite to each other and their sign only depends on the sign of $j_{1} j_{2}$. Thus, for a band structure with two bands with the same curvature, odd-frequency pairing always has a positive or diamagnetic Meissner effect from intra-band processes, while inter-band processes always give a negative or paramagnetic Meissner effect. On the other hand, for an inverted band structure, where then $j_{1} j_{2}<0$, we get the opposite behavior: intraband processes always generate a paramagnetic Meissner effect, while interband processes always give a diamagnetic effect.

To further analyze the results, especially establishing the relative sizes of the even- and odd-frequency and intra- and interband contributions to the Meissner effect, we need to perform the summation over reciprocal space in Eq. (12). Having established the importance of the sign of the product
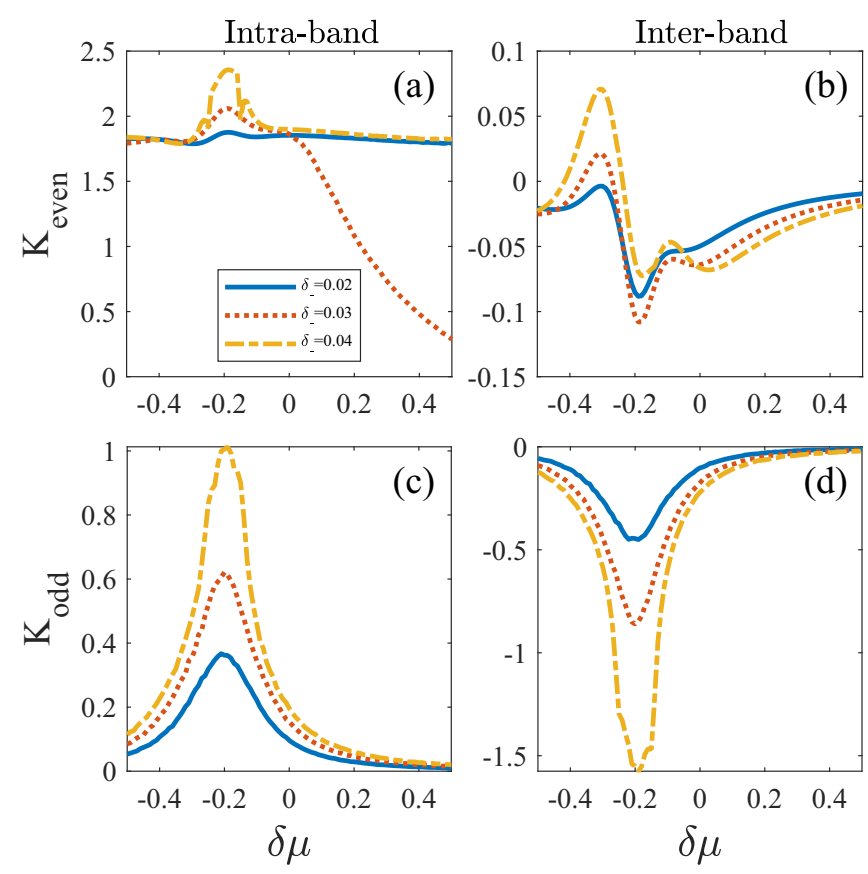

FIG. 2. Meissner effect divided into even- and odd-frequency (rows) and intra- and interband (column) contributions as a function of energy difference between orbitals $\delta \mu$ and for several values of orbital pairing asymmetry $\delta_{-}$. Here $t_{2}=0.5, t_{12}=0.05, \mu=0.3$, and $\delta_{+}=0.03$.

$j_{1} j_{2}$ in Eq. (13), we divide our numerical results into two distinct cases: two electronlike bands where $j_{1} j_{2}>0$ (or, equivalently, two holelike bands) and an inverted band structure with $j_{1} j_{2}<0$. In particular, below we choose $t_{2}= \pm 0.5$ and also set $\delta_{+}=0.03$ and $\xi_{12}$ to a $k$-independent constant $t_{12}$. We then vary all other parameters; $\mu, \delta \mu$, and $\delta_{-}$, to arrive at a comprehensive picture of the Meissner effect in generic two-orbital superconductors. In terms of determining the impact of odd-frequency pairing on the Meissner effect, it is particularly important to vary $\delta_{-}$as the odd-frequency pair amplitude is directly proportional to this pairing asymmetry, or orbital selectivity.

\section{A. Two electronlike bands, $j_{1} j_{2}>0$}

We begin with a material consisting of two low-energy electron-like bands, here generically modeled with $t_{1}=1$ and $t_{2}=0.5$, such that $j_{1} j_{2}>0$. In this case, the odd-frequency pairing generates an intra-band Meissner effect that is always diamagnetic, while the inter-band part is always paramagnetic, as given by Eq. (15). However, as we in the plots see below, even-frequency pairing generate terms which can change sign depending on parameter values.

We start by investigating in Fig. 2 the even- and oddfrequency contributions to the Meissner effect divided up into intra- and interband processes and as a function of the energy difference between orbitals $\delta \mu$, for different values of orbital selective superconducting order parameter $\delta_{-}$. As we fix $\delta_{+}=0.03$, we here investigate three different regimes. For $\delta_{+}>\delta_{-}$, we are in a so-called $s_{++}$phase where the phase of the superconducting order parameter on each orbital is the 
same: $\delta_{1,2}>0$, while for $\delta_{+}<\delta_{-}$we are in a $s_{+-}$phase where $\delta_{1}>0$ but $\delta_{2}<0$. At the boundary, $\delta_{-}=\delta_{+}=0.03$, we find $\delta_{2}=0$, i.e., only orbital 1 is intrinsically superconducting. Moreover, we set $\mu=0.3$, which makes the Fermi level cross both bands, see Fig. 1(a), and thus both bands give large contributions to the Meissner effect.

In Figs. 2(a) and 2(b), we plot the contributions from the even-frequency pairing to the Meissner response. The contribution from intraband processes [Fig. 2(a)] is completely dominating and remains positive for the full range of parameters values, i.e., a diamagnetic Meissner response. The behavior is more or less the constant for both $s_{++}$and $s_{+-}$ orbital pairing. The only exception is the special case $\delta_{+}=$ $\delta_{-}$, which develops a notable decrease when $\delta \mu$ is increasing. We can understand this behavior by noting that, in this case, there is no intrinsic superconductivity in orbital $2\left(\delta_{2}=0\right)$ and increasing $\delta \mu$ shifts the band bottom of orbital 1 to higher energies, thus making its Fermi surface smaller. As a consequence, the Meissner intraband contributions goes down with increasing $\delta \mu$. While the even-frequency interband contribution in Fig. 2(b) is much smaller, we note that it interestingly has no fixed sign. Turning to the odd-frequency contributions, we see in Figs. 2(c) and 2(d) that the intra- (inter)band part is dia- (para)magnetic, as also established by Eq. (15). Furthermore, we find that the interband contribution is generally larger than the intra-band contribution, in contrast to the dominating intraband process for the even-frequency pairing. This leaves the total Meissner effect from odd-frequency pairing paramagnetic, in agreement with the traditional expectation for the odd-frequency response [23-30], but we note that the overall effect is partly canceled due to a relatively large diamagnetic intraband contribution. Moreover, we find that the Meissner response from the odd-frequency pairing is significantly increasing with increasing orbital asymmetry $\delta_{-}$, in agreement with the effectively linear dependence on $\delta_{-}$for the odd-frequency pair amplitude.

In Fig. 3, we continue to analyze the Meissner response, focusing especially on how the different components compare to each other, with even-frequency intraband (blue), evenfrequency interband (red), odd-frequency intraband (yellow), and odd-frequency interband (purple) together with the total response (green). Based on the distinct behaviors observed in Fig. 2, we here focus on the overall dependence on the chemical potential [Figs. 2(a) and 2(b)], and the superconducting asymmetry $\delta_{-}$, as they determine the Fermi surface size and magnitude of the odd-frequency pairing, respectively. For the Meissner effect as a function of chemical potential, we choose to report values for both the $s_{++}$(a) and $s_{+-}$(b) regimes. We here see how all terms increase as a function of $\mu$, which is due to the Fermi surface increasing with $\mu$ and thus creating a stronger superconducting state, which is then reflected in an increase in the total Meissner effect. The only minor exception to this increasing trend is the even-frequency intraband contribution around $\mu \sim 0.22$ for $s_{+-}$pairing. Analyzing the superconducting order parameters in the band basis, we find that one of the intraband order parameters $\delta_{+}-\delta_{-} \cos (\theta)$ becomes zero at this particular point, thus causing the drop in the intraband Meissner effect (for details and definition of $\theta$, see Appendix B). This drop does not exist in Fig. 2(a) because there $\delta_{+}>\delta_{-}$and hence $\delta_{+}-\delta_{-} \cos (\theta)$ never becomes zero.
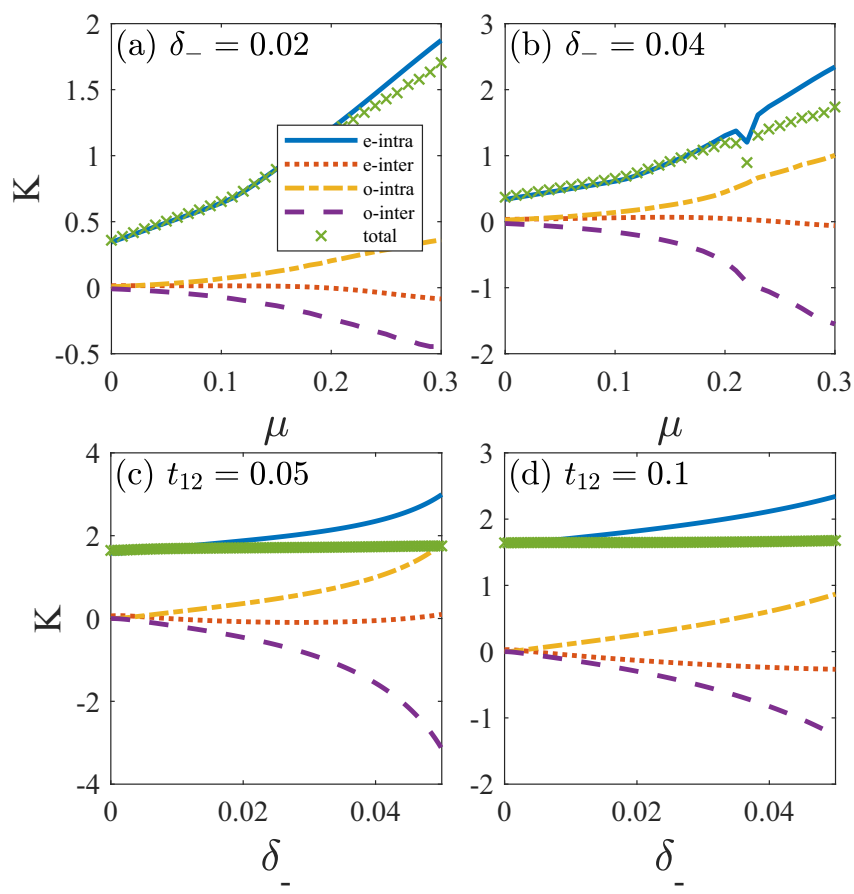

FIG. 3. Meissner effect as a function of overall chemical potential $\mu[(\mathrm{a}),(\mathrm{b})]$ and superconducting orbital asymmetry $\delta_{-}$ [(c), (d)] divided into even-frequency intraband (blue solid), evenfrequency interband (red dotted), odd-frequency intraband (yellow dash-dotted), and odd-frequency interband (purple dashed) contributions, together with the total effect (green $\times$ marks). Here $t_{2}=$ $0.5, \delta \mu=-0.2, \delta_{+}=0.03$, and $t_{12}=0.05[(\mathrm{a}),(\mathrm{b})]$ and $\mu=0.3$ $[(\mathrm{c}),(\mathrm{d})]$, with remaining parameters given in the figure panels.

We also find in Figs. 3(a) and 3(b) that both the intra- and interband odd-frequency contributions become larger when $\delta_{-}$increases. This increase is further verified in Figs. 3(c) and $3(d)$, where we plot the different contributions to the Meissner effect as a function of the superconducting asymmetry $\delta_{-}$. Here, we choose two different values of the interorbital hybridization $t_{12}=0.05$ [Fig. 3(c)] and 0.1 [Fig. 3(d)], with all other parameters similar to Figs. 3(a) and 3(b). All Meissner contributions increase as a function of $\delta_{-}$, but notably the odd-frequency terms increases faster. This is to be expected as the odd-frequency pair amplitude is linearly proportional to $\delta_{-}$, see Eq. (6). Note, however, that we do not find that the odd-frequency Meissner contributions increase in the same manner for the orbital hybridization $t_{12}$, despite this term also being necessary to generate odd-frequency pair amplitude $F^{o}$, see Eq. (6). This is due to the term $t_{12}$ also changing the whole band structure and thus the pair amplitude (through the denominator $D$ ) and the Meissner response are influenced rather substantially. We find numerically a maximum odd-frequency Meissner response around $t_{12} \sim 0.05$, see Appendix A. With $\delta_{-}$being a much smaller energy scale, the same band-structure effects are not seen for realistic $\delta_{-}$. Interestingly, we find that for the odd-frequency part, the interband contributions are larger than the intraband ones for all parameters in Fig. 3. However, the difference is always small, so, although the interband contribution is paramagnetic, the diamagnetic intraband contribution makes the total paramagnetic 
Meissner effect from the odd-frequency pairing quite small. As a consequence, odd-frequency pairing has only a minor effect on the Meissner effect in two-orbital superconductors with two electronlike (or holelike) bands. In fact, we find that the total Meissner effect is essentially constant when $\delta_{-}$ increases, despite this causing a strong increase in both oddfrequency pair amplitude and its influence on the Meissner effect. Hence we conclude that multiorbital superconductors are highly stable in a magnetic field, even when substantial bulk odd-frequency pairing is present. We emphasize that this is not due to small contributions to the Meissner effect from the odd-frequency pairing, but the fact that the intra- and interband odd-frequency processes nearly cancel each other.

\section{B. Two inverted bands, $j_{1} j_{2}<0$}

We next turn to the case of an inverted band structure, such that $t_{1} t_{2}<0$, and equivalently $j_{1} j_{2}<0$, as schematically illustrated in Fig. 1(b) and typical for topological materials [37]. This band structure never has both bands crossing the Fermi level, and there also needs to be a finite doping to reach beyond the insulating state. In this case, Eq. (15) shows that the odd-frequency pairing always gives a paramagnetic Meissner effect for intraband processes and a diamagnetic Meissner effect for the interband processes, i.e., opposite to the behavior in the previous section with a noninverted band structure. This analytical result is in agreement with what has been found earlier for the highly unconventional nematic interorbital spin-triplet superconducting state found in doped topological insulators [9], indicating that these signs of the Meissner effect contributions are likely stable for a range of different symmetries for $\hat{\Delta}$, even though we here are concentrating on the more common conventional intra-orbital spin-singlet $s$-wave superconducting state.

Proceeding with numerical results, we next report similar plots for the inverted band structure as we did for two electronlike bands in Figs. 2 and 3. Thus, in Fig. 4 we show the even- and odd-frequency (rows) and intra- and interband (columns) contributions to the Meissner effect as a function of $\delta \mu$. We here initially fix the chemical potential $\mu=0$ such that both bands contribute similarly at $\delta \mu=0$, but we check the $\mu$ dependence in the next figure. Again we choose three increasing values of $\delta_{-}$creating $\delta_{1} \delta_{2}>0\left(s_{++}\right), \delta_{2}=0$, and $\delta_{1} \delta_{2}>0\left(s_{+-}\right)$orbital pairing, respectively. Here we find that all Meissner contributions increases rapidly when $\delta \mu$ becomes more negative until around $\delta \mu \sim-0.3$. This is the value when the Fermi level crosses into the valence band, and thus this increase is due to more low-energy states being available. For even more negative values of $\delta \mu$ we see different trends. The even-frequency intraband contribution (a) continues to increase, as expected for a metallic state with an increasing Fermi surface. The only exception is the case of $\delta_{-}=0.04$, where, in a very limited regime, we surprisingly find a negative, paramagnetic contribution. This is the regime where superconductivity in one of the bands, $\delta_{+}-\delta_{-} \cos (\theta)$, approaches zero, see Appendix B. The even-frequency interband contribution (b) gives both diamagnetic and paramagnetic responses, dependent on the choice of parameters, and is now notably larger than in the previous case with $j_{1} j_{2}>0$ in Fig. 2. However, for the even-frequency
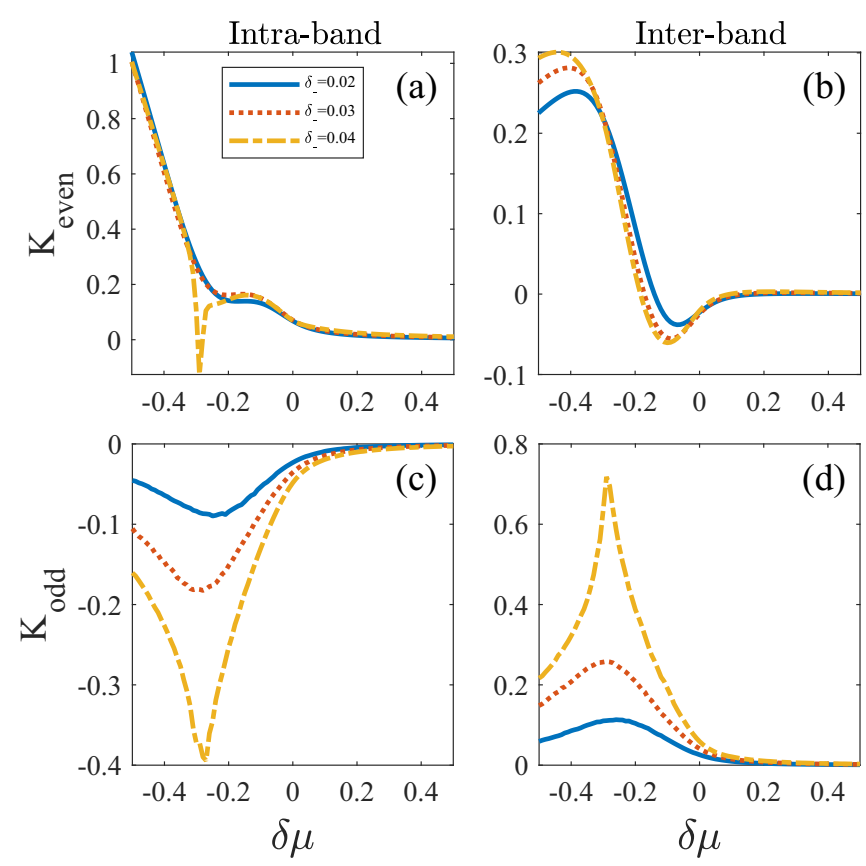

FIG. 4. Meissner effect divided into even- and odd-frequency (rows) and intra- and interband (columns) contributions as a function of energy difference between orbitals $\delta \mu$ and for several values of orbital pairing asymmetry $\delta_{-}$. Here $t_{2}=-0.5, t_{12}=0.05, \mu=0$, and $\delta_{+}=0.03$.

contribution, the intraband processes are still generally dominating. When it comes to the odd-frequency contributions to the Meissner effect, we see how they form a peak structure quite similarly to that of Fig. 2, indicating a general behavior of the odd-frequency contributions. Most importantly, here the diamagnetic interband contribution is much larger than the paramagnetic intraband contribution, which leaves the odd-frequency pairing giving a diamagnetic contribution to the total Meissner response. Thus, odd-frequency pairing actually helps in stabilizing the superconducting state through the generation of an increased diamagnetic Meissner effect, in complete contrast to many other systems where an odd-frequency paramagnetic Meissner effect has often been discussed [23-30].

Finally, in Fig. 5 we plot in the same subpanels all contributions to the Meissner effect as a function of chemical potential $\mu$ [Figs. 5(a) and 5(b)] and superconducting orbital asymmetry $\delta_{-}$[Figs. 5(c) and 5(d)], i.e., similarly to Fig. 3 but now for the inverted band structure. For small values of the chemical potential, the system is an insulator in the normal state and we find that many contributions to the Meissner effect are often of similar size. Interestingly, as we increase $\delta_{-}$by comparing Figs. 5(a) and 5(b), we get a notably higher contribution from the odd-frequency pairing and the odd-frequency inter-band part even becomes the clearly dominant contribution in this low $\mu$ regime. However, by increasing the chemical potential to larger values, the conventional even-frequency intraband part increases and eventually becomes dominant, with the total Meissner response now instead approaching its value. The transition between these two regimes is marked by the system going into a metallic regime around $\mu \sim 0.08$. It is 

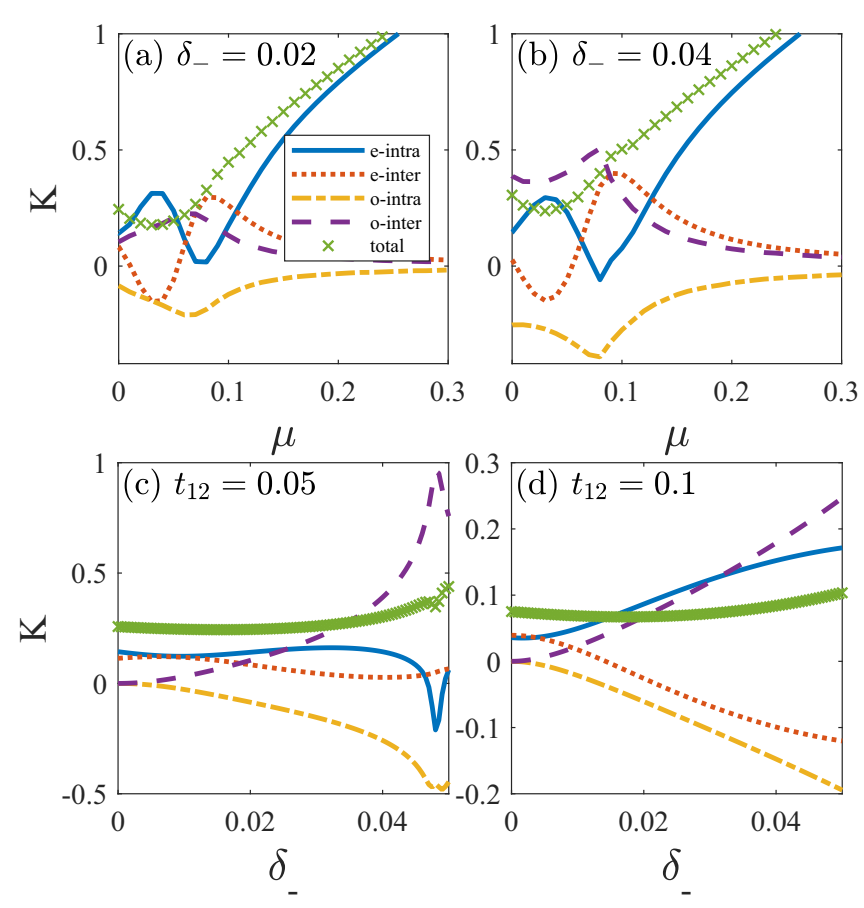

FIG. 5. Meissner effect as a function of overall chemical potential $\mu[(\mathrm{a}),(\mathrm{b})]$ and superconducting orbital asymmetry $\delta_{-}$ [(c), (d)] divided into even-frequency intraband (blue solid), evenfrequency inter-band (red dotted), odd-frequency intraband (yellow dash-dotted), and odd-frequency interband (purple dashed) contributions, together with the total effect (green $\times$ marks). Here $t_{2}=-0.5$, $\delta \mu=-0.2, \delta_{+}=0.03$, and $t_{12}=0.05[(\mathrm{a}),(\mathrm{b})]$ and $\mu=0[(\mathrm{c}),(\mathrm{d})]$, with remaining parameters given in the figure panels.

thus not surprising that interband processes are dominating in the insulating and near-metallic regime, $\mu<0.08$. Thus we find that the odd-frequency contributions to the Meissner effect is completely dominating in the insulating and nearmetallic regimes. Notably, the total Meissner effect is always diamagnetic, even with dominating odd-frequency contributions as they are also diamagnetic.

To further investigate the behavior of the Meissner effect when it is dominantly coming from the odd-frequency pairing, we plot in Figs. 5(c) and 5(d) the Meissner contributions as a function of $\delta_{-}$for $\mu=0$. These subpanels clearly show how, by increasing the $\delta_{-}$term both the intra- and inter-band contributions to the odd-frequency Meissner effect become large, especially for moderate $t_{12} \sim 0.05$ (c). While the intra-band contribution is paramagnetic, it is much smaller than the diamagnetic inter-band contribution, making the odd-frequency Meissner contribution diamagnetic and for larger $\delta_{-}$even clearly dominating the total Meissner response, see Appendix $\mathrm{C}$ for an additional plot where we sum the odd- and evenfrequency contributions to the Meissner response. Based on these results, we conclude that for inverted, or topological, band structures, odd-frequency pairing can easily dominate the Meissner response, especially at low doping levels in the near-metallic regime. It is interband odd-frequency processes that dominate in this regime and they always contribute diamagnetically to the Meissner effect. As a consequence, it is only the large odd-frequency pairing that stabilizes the superconducting state in a magnetic field.

\section{CONCLUSIONS}

Odd-frequency pairing has often been discussed to give a paramagnetic Meissner effect, with only conventional evenfrequency pairing assume to give the diamagnetic Meissner effect necessary to stabilize a superconductor in an external magnetic field [23-30]. As a result, the existence of large odd-frequency pair amplitudes has been thought to destabilize superconductivity, possibly even to the degree where it cannot exist as a bulk effect [28]. Here we disprove this simplistic picture for multiorbital superconductors where odd-frequency bulk superconducting pairing is ubiquitous. We show that odd-frequency pairing actually generates both dia- and paramagnetic contributions, and that they usually either nearly cancel each other or even generate an overall diamagnetic Meissner effect. As a result, even large odd-frequency pairing in the bulk does not destabilize superconductivity in multiorbital superconductors.

In more detail, we are able to derive, using only a few nonrestrictive assumptions, simple analytical results for the Meissner response in a generic two-orbital superconductor, with a standard quadratic normal-state dispersion and conventional $s$-wave superconductivity. In these two-orbital superconductors, odd-frequency pairing is always present as soon as there exists a finite orbital hybridization and an asymmetry in the superconducting pairing between the two orbitals, with the odd-frequency pair amplitude growing linearly with the latter. By dividing up the Meissner effect into contributions from even- and odd-frequency pairing, as well as intraand interband processes, we show analytically that for oddfrequency pairing, intra- and interband processes contribute with different signs to the Meissner effect, with the sign directly tied to the character of the normal-state band structure.

For two electronlike (or holelike) bands (i.e., same sign of the quadratic curvature in both bands) we always find that the odd-frequency pairing gives diamagnetic intraband but paramagnetic interband contributions to the Meissner effect. Numerically we further show that, while this interband part is usually slightly larger, the two contributions nearly cancel in all relevant parameter regimes. Together with a relatively large even-frequency diamagnetic Meissner response, this results in an overall stabilizing diamagnetic Meissner effect, even in the limit of large odd-frequency pairing in the superconductor. In contrast, for an inverted band structure (i.e.. with different signs of the quadratic curvatures in the two bands, as, e.g., found in topological materials), we instead always find that the odd-frequency pairing gives diamagnetic interband but paramagnetic intraband contributions. This particular result is in agreement with earlier findings for the nematic superconducting phase in doped topological insulators [9], illustrating how the result is likely also stable in the presence of strong spin-orbit coupling or unconventional superconducting pairing. Very interestingly, the odd-frequency interband contribution even becomes the dominating contribution to the total Meissner effect in the near-metallic regime where the Fermi level is close to the valence or conduction band bottom. Thus, the Meissner effect can even be completely 
driven by the odd-frequency pairing, but still be diamagnetic. Moreover, for both types of band structures, we find that the even-frequency contributions to the Meissner effect is often diamagnetic but can actually become paramagnetic for some parameter ranges. Still, we always find a stable diamagnetic Meissner effect, even in superconductors with large odd-frequency bulk pairing.

To summarize, our results show that bulk odd-frequency bulk pairing does not cause a destabilizing paramagnetic Meissner effect in multiorbital superconductors. Instead, oddfrequency pairing either does not contribute significantly to the Meissner effect or, very interestingly, gives a diamagnetic contribution in topological band structures. Based on this diamagnetic odd-frequency Meissner response in topological materials, we speculate that odd-frequency pairing might be quite common in a wide range of topological materials.

\section{ACKNOWLEDGMENTS}

The authors thank J. Schmidt and D. Chakraborty for fruitful discussions and acknowledge financial support from the European Research Council (ERC) under the European Unions Horizon 2020 research and innovation program (No. ERC-2017-StG-757553) and the Knut and Alice Wallenberg Foundation through the Wallenberg Academy Fellows program.

\section{APPENDIX A: MOMENTUM-DEPENDENT INTERORBITAL HYBRIDIZATION}

An assumption made in the main text was to assume a constant hybridization between the two orbitals: $\xi_{12}=t_{12}$. This offers a significant simplification as it gives a current operator of the form $\left(\begin{array}{cc}j_{1} & 0 \\ 0 & j_{2}\end{array}\right)$, which is needed to proceed with the analytical calculations. Without this assumption, we could not derive the relatively simple expressions in Eqs. (14) and (15) for the Meissner effect from even- and odd-frequency pairing. In this Appendix, we first present the behavior of Meissner effect with respect to $t_{12}$ to provide a comprehensive view of the dependence on the orbital hybridization, and then we present results for a momentum-dependent hybridization $\xi_{12}(k)$, showing that such a change does not qualitatively change our conclusions.

In Fig. 6, we plot the behavior of the even- and oddfrequency and intra- and interband contributions to the Meissner effect as a function of the $k$-independent orbital hybridization $t_{12}$. We further set $\delta_{+}=0.03, \delta_{-}=0.04, \delta \mu=$ -0.2 and use in (a) an electronlike band structure with $t_{2}=$ 0.5 and $\mu=0.3$ and in (b) an inverted band structure with $t_{2}=-0.5$ and $\mu=0.0$ to comply with the parameter choices in the main text. These superconducting order parameters render a $s_{+-}$state with substantial odd-frequency pairing due to the large size of $\delta_{-}$. The results show that for the region $t_{12} \lesssim 0.1$, all different contributions to the Meissner effects are reasonably large, while for larger $t_{12}$ the even-frequency intraband contribution becomes dominant. This motivates our choice of $t_{12} \leqslant 0.1$ in the main text to capture the most interesting regime. Still, all conclusions drawn in the main text, in terms of both magnitudes and signs of the Meissner effect, hold true for the full range of $t_{12}$.

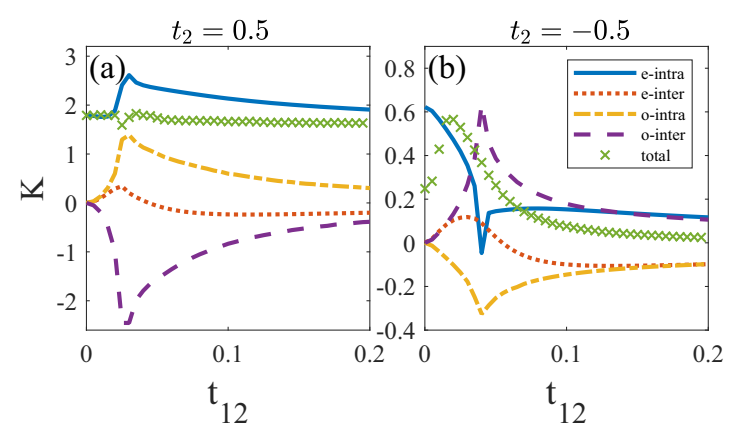

FIG. 6. Meissner effect as a function of orbital hybridization $t_{12}$ divided into even-frequency intraband (blue solid), even-frequency interband (red dotted), odd-frequency intraband (yellow dashdotted), and odd-frequency interband (purple dashed) contributions, together with the total effect (green $\times$ marks) for (a) electronlike band structure with $t_{2}=0.5, \mu=0.3$ and (b) inverted band structure with $t_{2}=-0.5, \mu=0$. Here $\delta \mu=-0.2, \delta_{+}=0.03$, and $\delta_{-}=0.04$, creating a $s_{+-}$state.

Next we investigate how the Meissner response changes if we instead use a momentum-dependent orbital hybridization, $\xi_{12}(k)$. In Fig. 7, we show the different contributions to the Meissner effect as a function of the orbital energy difference $\delta \mu$ for a $k$-dependent hybridization between orbitals of the
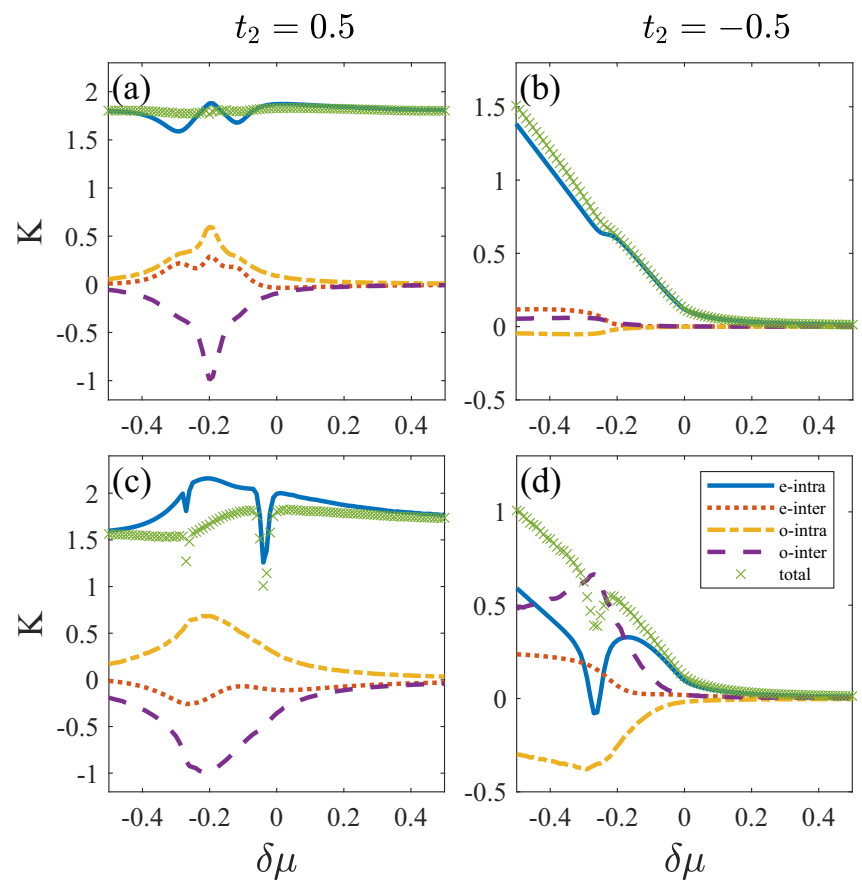

FIG. 7. Meissner effect as a function of orbital energy difference $\delta \mu$ divided into even-frequency intraband (blue solid), even-frequency interband (red dotted), odd-frequency intraband (yellow dash-dotted), and odd-frequency interband (purple dashed) contributions, together with the total effect (green $\times$ marks) for (a), (c) electronlike band structure with $t_{2}=0.5, \mu=0.3$ and (b), (d) inverted band structure with $t_{2}=-0.5, \mu=0$ and a momentumdependent orbital hybridization $\xi_{12}=t_{12} k^{2}$, with (a), (b) $t_{12}=0.05$, and (c), (d) $t_{12}=0.2$. Here $\delta_{+}=0.03$ and $\delta_{-}=0.04$, creating a $s_{+-}$ state. 


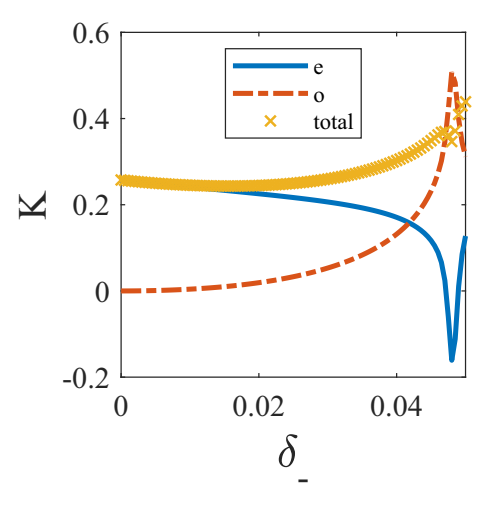

FIG. 8. Meissner effect as a function superconducting orbital asymmetry $\delta_{-}$divided into the total even-frequency (blue solid) and odd-frequency (red dash-dotted) contribution, together with the total effect (yellow $\times$ marks). Here $t_{2}=-0.5, \delta \mu=-0.2, \delta_{+}=0.03$, $t_{12}=0.05$, and $\mu=0$ in analogy with Fig. 5(c).

form $\xi_{12}(k)=t_{12} k^{2}$. We further set $\delta_{+}=0.03, \delta_{-}=0.04$, creating a $s_{+-}$state and report results for both an electronlike band structure [Figs. 7(a) and 7(c)] with $t_{2}=0.5, \mu=0.3$, and an inverted band structure [Figs. 7(b) and 7(d)] with $t_{2}=$ $-0.5, \mu=0.0$. We also vary the hybridization strength, with $t_{12}=0.05$ [Figs. 7(a) and 7(b)] and $t_{12}=0.2$ [Figs. 7(c) and $7(d)]$. The results in these figures are in very good agreement with figures in the main text, Figs. 2 and 4, regarding the sign and magnitude of different contributions to the Meissner effect, and thus do not qualitatively change any of our conclusions. Exactly the same as in the main text, the odd-frequency intra- and interband contributions to the Meissner effect have

$$
\hat{H}_{b}=\left(\begin{array}{cc}
\epsilon_{+} & 0 \\
0 & \epsilon_{-} \\
\delta_{+}+\delta_{-} \cos (\theta) & -2 \delta_{-} \cos ^{2}\left(\frac{\theta}{2}\right) \\
-2 \delta_{-} \sin ^{2}\left(\frac{\theta}{2}\right) & \delta_{+}-\delta_{-} \cos (\theta)
\end{array}\right.
$$

The Hamiltonian $H_{b}$ in the band basis nicely illustrates two features. First, the only coupling between the two bands is through the interband pairing terms, proportional to $\delta_{-}$. Thus, in the $\delta_{-} \rightarrow 0$ limit, the superconducting part of the Hamiltonian $H_{b}$ would commute with the normal part. As a result, odd-frequency pairing is a direct consequence of this interband term [7]. This shows that odd-frequency pairing from the interband pairing is fundamentally the same and only differs with a basis change from the odd-frequency pairing generated by orbital hybridization $\xi_{12}$ and orbital pairing asymmetry $\delta_{-}$ used in the main text. Second, the intraband pairing terms becomes explicit as $\delta_{+} \pm \delta_{-} \cos (\theta)$ and it is easy to see when one of the bands become nonsuperconducting, a result used in the main text to explain some of the even-frequency intraband results.

$$
\left.\begin{array}{cc}
\delta_{+}+\delta_{-} \cos (\theta) & -2 \delta_{-} \cos ^{2}\left(\frac{\theta}{2}\right) \\
-2 \delta_{-} \sin ^{2}\left(\frac{\theta}{2}\right) & \delta_{+}-\delta_{-} \cos (\theta) \\
-\epsilon_{+} & 0 \\
0 & -\epsilon_{-}
\end{array}\right) .
$$

opposite signs with respect to each other and also inverting the band structure flips the signs of these two contributions. Due to the opposite signs of the intra- and interband contributions, the total Meissner contribution from odd-frequency pairing is always small and hence even large odd-frequency pairing never produces a destabilizing Meissner response, even for a momentum-dependent orbital hybridization. Additionally, we find for the case $t_{12}=0.05$ that the odd-frequency contribution is relatively small, but it increases when we use $t_{12}=0.2$ in Figs. 7(c) and 7(d). This can easily be understood from the fact that the $t_{12}$ term is now multiplied by a $k^{2}$ dispersion, which reduces its effect at low momenta. Hence, to get a similar behavior to that of the $k$-independent case with $\xi_{12}=t_{12}$, we need to use larger values of prefactor $t_{12}$.

\section{APPENDIX B: BAND BASIS HAMILTONIAN}

In this Appendix, we transform the two-orbital Hamiltonian in Eq. (2) into the band basis where the kinetic energy is fully diagonal. While the original orbital basis of Eq. (2) is convenient for a lot of the derivations in the main text, the band basis gives a better intuition for some of our results.

The normal-state Hamiltonian in the main text, $h(k)=$ $\xi_{+} \tau_{0}+\xi_{-} \tau_{z}+\xi_{12} \tau_{x}$, is diagonalized and thus transformed into the band basis with the rotation matrix

$$
\hat{R}_{\theta / 2}=\left(\begin{array}{cc}
\cos (\theta / 2) & -\sin (\theta / 2) \\
\sin (\theta / 2) & \cos (\theta / 2)
\end{array}\right)
$$

where $\theta=\sin ^{-1}\left(\xi_{12} / \sqrt{\xi_{12}^{2}+\xi_{-}}\right)$. Applying this rotation matrix also to the superconducting part of Eq. (2), we retrieve the Hamiltonian in the band basis as

\section{APPENDIX C: DOMINATING ODD-FREQUENCY CONTRIBUTION}

To visually compare the total contributions from evenand odd-frequency pairing to the Meissner contributions in Fig. 5 we need to sum up the contributions from interband and intraband processes for both types of pairing. In Fig. 8, we present such summation for the plot in Fig. 5(c), along with the total Meissner effect. The figure shows a range of $\delta_{-}$, for which the contributions from the odd-frequency pairing clearly dominates that of the even-frequency pairing. Interestingly, for a small range of $\delta_{-}$the even-frequency contribution even becomes negative and it is thus the oddfrequency contribution which makes the system overall diamagnetic.
[1] V. L. Berezinskii, Pis'ma Zh. Eksp. Teor. Fiz. 20, 628 (1974) [JETP Lett. 20, 287 (1974)].
[2] J. Linder and A. V. Balatsky, Rev. Mod. Phys. 91, 045005 (2019). 
[3] J. Cayao, C. Triola, and M. A. Black-Schaffer, Eur. Phys. J.: Spec. Top. 229, 545 (2020).

[4] F. S. Bergeret, A. F. Volkov, and K. B. Efetov, Rev. Mod. Phys. 77, 1321 (2005).

[5] F. S. Bergeret, A. F. Volkov, and K. B. Efetov, Phys. Rev. Lett. 86, 4096 (2001).

[6] A. F. Volkov, F. S. Bergeret, and K. B. Efetov, Phys. Rev. Lett. 90, 117006 (2003).

[7] C. Triola, J. Cayao, and A. M. Black-Schaffer, Ann. Phys. 532, 1900298 (2020).

[8] A. M. Black-Schaffer and A. V. Balatsky, Phys. Rev. B 88, 104514 (2013).

[9] J. Schmidt, F. Parhizgar, and A. M. Black-Schaffer, Phys. Rev. B 101, 180512(R) (2020).

[10] P. Burset, B. Lu, H. Ebisu, Y. Asano, and Y. Tanaka, Phys. Rev. B 93, 201402(R) (2016).

[11] B. Sothmann, S. Weiss, M. Governale, and J. König, Phys. Rev. B 90, 220501(R) (2014).

[12] F. Parhizgar and A. M. Black-Schaffer, Phys. Rev. B 90, 184517 (2014).

[13] E. M. Nica, R. Yu, and Q. Si, npj Quantum Mater. 2, 24 (2017).

[14] F. Parhizgar and A. M. Black-Schaffer, Sci. Rep. 7, 9817 (2017).

[15] L. Komendová and A. M. Black-Schaffer, Phys. Rev. Lett. 119, 087001 (2017).

[16] C. Triola and A. M. Black-Schaffer, Phys. Rev. B 97, 064505 (2018).

[17] F. Parhizgar and A. M. Black-Schaffer, npj Quantum Mater. 5, 42 (2020).

[18] P. Dutta, F. Parhizgar, and A. M. Black-Schaffer, Phys. Rev. B 101, 064514 (2020).

[19] Y. Tanaka, Y. Tanuma, and A. A. Golubov, Phys. Rev. B 76, 054522 (2007).
[20] M. Eschrig and T. Löfwander, Nat. Phys. 4, 138 (2008).

[21] Y. Asano, Y. Tanaka, and A. A. Golubov, Phys. Rev. Lett. 98, 107002 (2007).

[22] P. Dutta and A. M. Black-Schaffer, Phys. Rev. B 100, 104511 (2019).

[23] E. Abrahams, A. Balatsky, D. J. Scalapino, and J. R. Schrieffer, Phys. Rev. B 52, 1271 (1995).

[24] T. Yokoyama, Y. Tanaka, and N. Nagaosa, Phys. Rev. Lett. 106, 246601 (2011).

[25] M. Alidoust, K. Halterman, and J. Linder, Phys. Rev. B 89, 054508 (2014).

[26] Y. V. Fominov, Y. Tanaka, Y. Asano, and M. Eschrig, Phys. Rev. B 91, 144514 (2015).

[27] A. Di Bernardo, Z. Salman, X. L. Wang, M. Amado, M. Egilmez, M. G. Flokstra, A. Suter, S. L. Lee, J. H. Zhao, T. Prokscha, E. Morenzoni, M. G. Blamire, J. Linder, and J. W. A. Robinson, Phys. Rev. X 5, 041021 (2015).

[28] S. Hoshino, Phys. Rev. B 90, 115154 (2014).

[29] S.-P. Lee, R. M. Lutchyn, and J. Maciejko, Phys. Rev. B 95, 184506 (2017).

[30] J. A. Krieger, A. Pertsova, S. R. Giblin, M. Döbeli, T. Prokscha, C. W. Schneider, A. Suter, T. Hesjedal, A. V. Balatsky, and Z. Salman, Phys. Rev. Lett. 125, 026802 (2020).

[31] W. Meissner and R. Ochsenfeld, Naturwissenschaften 21, 787 (1933).

[32] R. Heid, Z. Phys. B: Condens. Matter 99, 15 (1995).

[33] H. Kusunose, Y. Fuseya, and K. Miyake, J. Phys. Soc. Jpn. 80, 044711 (2011).

[34] A. Ramires and M. Sigrist, Phys. Rev. B 94, 104501 (2016).

[35] T. Mizoguchi and M. Ogata, J. Phys. Soc. Jpn. 84, 084704 (2015).

[36] Y. Asano and A. Sasaki, Phys. Rev. B 92, 224508 (2015).

[37] M. Z. Hasan and C. L. Kane, Rev. Mod. Phys. 82, 3045 (2010). 